\title{
A commentary to the manuscript awareness and uptake of measures for preventing CNS birth defects among mothers of affected children in a sub-Saharan African neurosurgeon's practice
}

\author{
Ahmed Ammar ${ }^{1}$ \\ Received: 11 October 2015 / Accepted: 14 October 2015 /Published online: 24 October 2015 \\ (C) Springer-Verlag Berlin Heidelberg 2015
}

\section{Comment}

I would like to congratulate Dr. Amos Olufemi Adeleye, for writing the important article "Awareness and uptake of measures for preventing CNS birth defects among mothers of affected children in a sub-Saharan African neurosurgeon's practice" which I read with great interest. This article demonstrates clearly the low level of awareness of mothers about neural tubes defects, and the different methods for intrauterine screening and prevention of, or at least significant reduction of the risk of neural tube defects. This article supports previously similar studies conducted in Uganda [1] and Tanzania [2]

It is clear that problem extends to different sub-Saharan African countries. Therefore, it is important for different international organizations, continental, and national, governmental and non-governmental to get together to address the problem. There are several factors which need to be considered in order to produce a successful plan, which are the following:

1. The relatively young age of marriage in these countries.

2. The low income of several young mothers

3. The scattered health care and maternity care facilities.

4. Premarriage medical examination and screening may not exist or at least be unpopular

Therefore, facing the problem should not only be in hospital care facilities; it should include all components of the society including schools, media, churches, mosques, and public events to spread the knowledge and increase the awareness of mothers about the risk and sequel of neural tube defects (NTD).

The premarriage medical examination should be encouraged and should be made available for young ladies. These sessions of examination can be used to inform them about NTD and the importance of taking folic acid before getting pregnant. It is also important to repeatedly insist on the utmost importance of attending maternity care clinics and regular US examination of the fetus as early as the first few weeks of pregnancy. The option of termination of pregnancy in this early period should be available to every mother and should be made acceptable. I believe the doctors (Public health officials, obstetricians, pediatricians, neurosurgeons, and others) should be supported by their colleagues all over the world and reach the deep core of the society to increase the awareness of the society in general and young females in particular about NTD and different options to deal with the problem.

\section{References}

1. Femke B, Rita L, Peter K, Lieven B, van Hove G (2015) Prevention of spina bifida: folic acid intake during pregnancy in Gulu district, northern Uganda. Pan Afr Med J 20:90

2. Kinasha AD, Manji K (2002) The incidence and pattern of neural tube defects in Dar es Salaam, Tanzania. Eur J Pediatr Surg 12(1): S38-9

Ahmed Ammar

ahmed@ahmedammar.com

1 Al Khobar, Eastern Province, Saudi Arabia 